\title{
Transdermal Methimazole for Feline Hyperthyroidism
}

\author{
Elizabeth Hicks ${ }^{1}$ and Pilar Z Murphy ${ }^{2 *}$ \\ ${ }^{1}$ PharmD Graduate 2014, Samford University McWhorter School of Pharmacy, USA \\ ${ }^{2}$ Department of Pharmacy Practice, Samford University McWhorter School of Pharmacy, USA
}

Submission: July 17, 2019; Published: August 19, 2019

*Corresponding author: Pilar Z Murphy, PharmD, BCACP, Associate Professor of Pharmacy Practice, Samford University McWhorter School of Pharmacy, 800 Lakeshore Drive, Birmingham, AL 3529, USA

\begin{abstract}
Hyperthyroidism is a common disorder in older cats causing detrimental adverse effects if left untreated. The three most recommended treatment options include thyroidectomy, radioiodine treatment, and antithyroid medication therapy. Oral methimazole has been the most widely used option due to low cost and accessibility. The topical application of transdermal methimazole is an ideal route of administration for cat owners. The purpose of this review article is to give insight into the efficacy and recommended indication for use of the pluronic lecithin organogel (PLO) formulated transdermal delivery system of methimazole, in the treatment of feline hyperthyroidism. PLO compounded methimazole is uniquely transported through the skin, and chronic use has been shown effective in treating feline hyperthyroidism. In many cases, once daily application of the gel has provided enough methimazole activity for lowering hormone levels. The compounded formulation also allows for more individualized dosing than the oral tablets. There is limited information regarding long-term treatment of PLO methimazole, however, the formulation continues to satisfy both veterinarians and owners, and effectively lower serum thyroxine (T4) concentrations.
\end{abstract}

Keywords: Feline; Hyperthyroidism; Transdermal; Methimazole

Abbreviations: PLO: Pluronic Lecithin Organogel

\section{Introduction}

Over the past 20 years, the prevalence offeline hyperthyroidism has increased astoundingly [1-3]. It has become the most common endocrine disorder in cats, and the risk worsens with each year of increasing age, being most common in middle to older-aged felines [1]. The disease is primarily characterized by an excessive production and release of the thyroid hormones thyroxine (T4) and triiodothyronine (T3) most commonly due to a functional, benign adenomatous hyperplasia of the thyroid gland. At present, there is not a feline specific thyroid stimulating hormone (TSH) assay test available, therefore unlike human hyperthyroid

Table 1: Clinical Complications of Hyperthyroidism: Signs and Symptoms [6,10].

\begin{tabular}{|c|c|}
\hline \multicolumn{1}{|c|}{ Symptoms } & Signs \\
\hline Emotional lability, nervousness, irritability & Hyperactivity, rapid speech \\
\hline Muscle weakness & Fine, moist skin; fine, abundant hair \\
\hline Palpitations & Ophthalmopathy \\
\hline Polyphagia, weight loss & Tachycardia, atrial fibrillation, widened pulse pressure, dyspnea \\
\hline Hyperdefecation, polyuria & Goiter \\
\hline Heat intolerance & Menstrual changes \\
\hline
\end{tabular}

diagnosis, veterinarians do not commonly depend on a low TSH value for primary hyperthyroid diagnosis. Hyperthyroidism diagnosis in cats is generally based on a high free T4 level and the presence of clinical signs and symptoms. Some of the clinical complications of hyperthyroidism that may be present include emotional lability, hyperactivity, palpitations, tachycardia, and a plethora of other manifestations of the disease (Table 1). Although the exact etiology is unknown, many nutritional and environmental causes are suspected, including canned cat food products containing iodine, soybean, phthalates, polyphenols, and polychlorinated biphenyls $[2,4,5]$. 
Regardless of the etiologic origin, medical management of prolonged thyroid hormone elevation is very important. Untreated hyperthyroidism can have many consequences on the cat. Many cats initially present with a change in personality or behavior, often being more easily agitated and mean, as well as with unexplained weight loss, changes in eating habits, accelerated heart rates, and a goiter. Hyperthyroidism, if left untreated, can also have life threatening adverse effects, such as causing hypertension, cardiac tachyarrhythmia, atrial fibrillation, and even death [6,7]. These result from elevated thyroid hormone levels and cause up-regulation of various gene expressions involved in the body's metabolism, thermogenesis for heat regulation, nerve function, and muscle and bone function [7]. They also function to increase activation of the sympathetic nervous system, which elevates the heart rate, the heart's force of contraction, and increases cardiac output overall $[8,9]$. Clearly, both the symptoms of the disease, as well as the enhancement of these biochemical pathways, can pose serious health risks to the feline patient. The longer a cat goes without treatment, the worse their complications become $[6,10,11]$.

Like the management of hyperthyroidism in humans, there are several different treatment options available for cats. The top three recommended therapies include surgical thyroidectomy, radioiodine therapy, and medication treatment. Thyroidectomy and radioiodine treatment can be permanent solutions to the disease. However, limitations such as expense and permanent hypothyroidism prevent these from being practical options for most feline patients $[7,11]$ (Table 2). Medication therapy is often the most practical and accessible way to manage hyperthyroid cats. Methimazole (Tapazole, Felimazole) is the most common and favored agent in the United States [12]. Other alternatives include carbimazole (a prodrug of methimazole marketed only in the UK), iodine-containing agents, iodine dietary restricted food, and homeopathic regimens [6,12-14]. Dietary iodine restriction is another option, however, there is limited supporting data to determine a true benefit.

Table 2: Advantages and Disadvantages of Feline Hyperthyroidism Treatment Options [7,11].

\begin{tabular}{|c|c|c|}
\hline Treatment & Advantages & Disadvantages \\
\hline \multirow{5}{*}{ Radioactive iodine (131I) } & Most effective & High initial expense \\
\hline & Few and rare side effects & Limited availability and inconvenient \\
\hline & Curative & Irreversible \\
\hline & Single injection & Permanent hypothyroidism \\
\hline & Most cost effective & \\
\hline \multirow{8}{*}{ Thyroidectomy } & Rapid, effective treatment & Most costly \\
\hline & Effective in patients with goiters & Most invasive \\
\hline & Curative & Anesthesia risks \\
\hline & & Hypo-parathyroidism risk \\
\hline & & Nerve laryngeal damage risk \\
\hline & & Irreversible \\
\hline & & Permanent hypothyroidism \\
\hline & & Pain, scar \\
\hline \multirow{5}{*}{ Antithyroid drugs } & Low initial expense & Low cure rate \\
\hline & Noninvasive & Daily drug administration \\
\hline & Low risk of permanent hypothyroidism & Compliance issues \\
\hline & Effective in cats without side effects & Adverse Drug Reactions \\
\hline & Reversible & For short-term treatment only \\
\hline
\end{tabular}

Although several treatment options are available for hyperthyroid cats, each therapy option has considerable drawbacks to both the client and the feline patient. Oral methimazole has historically been the most accessible and affordable choice. However, gastrointestinal side effects and an unfavorable twice-a-day oral administration schedule often limit its ultimate therapeutic outcomes in the cat. Both negative attributes are avoided with use of the transdermal methimazole gel compound. Due to the limited amount of data available on transdermal methimazole, this review aims to evaluate whether the pluronic lecithin organogel (PLO) compound of methimazole is effective in treating hyperthyroid cats. In addition, it also serves to provide insight on the recommendations for its use.

\section{Methods}

A PubMed search was conducted to identify articles in which the safety or efficacy of transdermal methimazole for treatment of feline hyperthyroidism was assessed. Key MeSH search terms included feline hyperthyroidism with a subheading for treatment. In addition, feline hyperthyroidism plus one of the following search terms were searched: treatment, drug-related side effects 
and adverse drug reactions. A free-text search was also conducted to identify articles not included in the MeSH term search. Metaanalyses, randomized controlled clinical trials, and case reports were included in the review if the primary focus of the article related to the use of oral or transdermal methimazole for feline hyperthyroidism. Studies were excluded if published in languages other than English. In addition, studies highlighting mechanisms of action, studies of pharmacodynamics or pharmacokinetic effects were excluded.

\section{Results}

Clinical data on the topic of feline hyperthyroidism treatment is limited. A PubMed search revealed 14 articles with transdermal methimazole and feline hyperthyroidism as a subheading. Of the articles used in this review, there were six that directly assessed the use and efficacy of transdermal methimazole in the treatment of feline hyperthyroidism. Of those six, five were small clinical studies and one was a case report/series.

\section{Evaluation of oral methimazole}

Oral methimazole has remained the mainstay of feline hyperthyroidism treatment since the early 1980's. It reversibly suppresses thyroid hormone levels by inhibiting thyroid peroxidase. It does not inactivate circulating T4 and T3, resulting in a 2 to 4-week delay before serum T4 concentrations begin to normalize [8]. While it accumulates in the thyroid gland, it does not block the release of preformed hormone, nor does it help reduce goiters $[8,15]$. Oral methimazole has variable bioavailability ranging from 27 to $100 \%$ so its efficacy varies from patient to patient [6]. The recommended dose for maximum efficacy is $2.5 \mathrm{mg}$ administered twice daily.

In a randomized, unblinded, clinical trial by Trepanier et al. [11], forty methimazole naive cats with newly diagnosed hyperthyroidism were studied to compare the efficacy of one daily dosing of oral methimazole to twice daily dosing. Owners completed a questionnaire of their cat's baseline behavior status and reported any changes that occurred during the study. The overall efficacy of once daily methimazole was found to be less effective than twice daily dosing. Serum T4 concentrations were considerably higher in cats receiving once daily dosing, and only $54 \%(13 / 24)$ were found to be euthyroid at two weeks, compared to $87 \%(13 / 15)$ euthyroid in the twice daily group [16]. Both treatment groups showed considerable clinical improvement of many complications caused by hyperthyroidism. However, among the initial 40 cats studied, one cat in the once daily dosing group was removed prior to the 2-week point due to considerable gastrointestinal (GI) upset. Of the remaining 38 feline patients, 17 (44\%) developed some type of adverse event throughout the four-week duration. Throughout the remainder of the study, $23 \%$ (9 cats) reported similar GI upset. Among the 24 cats treated once daily, 42\% (10/24) required discontinuation of therapy, in order to resolve oral methimazole induced adverse events. Facial excoriation was reported in six patients, five reported from the once daily dosed group alone. Five of the six total facial excoriation cases reported were from the once daily dosed group. Manifestations of blood dyscrasias and hepatopathy were not significantly reported in either group [16].

Not only were adverse events such as GI upset and facial excoriations, found to be less prevalent in cats dosed twice a day, but also these cats were also more likely to obtain the goal euthyroid state. Cats also show rebound increases in serum T4 concentrations and a return to hyperthyroid state within 24 to 48 hours of methimazole discontinuation $[3,16,17]$. This likely correlates with the need for twice daily dosing in cats, and further research should be performed to help determine methimazole's true intrathyroidal residence time in cats. Oral methimazole is not a cure for feline hyperthyroidism, and treatment must be continued indefinitely. With the intolerable GI upset from the oral tablets and the difficulty many owners face administering the medication twice daily to uncooperative cats, the alternative transdermal route of administration poses significant benefits [16].

\section{Transdermal methimazole formulation}

Despite the limited clinical studies on transdermal methimazole, some clinicians have achieved a good therapeutic benefit to using this dosage form in cats. Pluronic lecithin organogel is a microemulsion-based gel containing lecithin, isopropyl palmitate, and pluronic acid to effectively deliver both hydrophilic and lipophilic drugs topically across the stratum corneum and may aid in the administration of methimazole [1822]. PLO is composed of both an oil phase (lecithin phase) and an aqueous phase (pluronic phase). It includes isopropyl palmitate acts as a solvent and permeation enhancer while lecithin also serves as a permeation enhancer by increasing the fluidity of the stratum corneum, and slightly disorganizing the skin structure to permit substance permeation [23-25]. PLO reversibly turns into a thick gel at body temperature, leading to an increase in dehydration of the aqueous solution, forming a shell-like structure of aggregated micelles [7,24-28]. Methimazole is an ideal drug for transdermal delivery due to its low molecular weight, high lipid solubility, water solubility, low daily dose, and is non-irritating and non-sensitizing to the skin [20,24].

\section{Efficacy of the PLO methimazole}

In a small retrospective study examining dispensing records for 16 hyperthyroid cats undergoing transdermal methimazole treatment, the transdermal formulation was effective at reducing serum T4 concentrations in 15 of the 16 cats studied. One cat showed an increase in serum T4 level, but there is no mention or clarification of appropriate application or other possible contributing factors. The only adverse event reported was a single case of increased blood urea nitrogen level, thought to be the unmasking of prior renal disease. This study also demonstrates variability in dosing and administration frequency of the topical, ranging between $5 \mathrm{mg}$ once a day to a twice daily dose of $7.5 \mathrm{mg}$ 
every morning and $5 \mathrm{mg}$ every night. This wide variation between each feline patient, limits our ability to recommend a standard dose or administration frequency, but does indicate the need for patient-specific doses and frequencies in order to effectively reach the euthyroid goal [29].

In a randomized clinical trial conducted by Sartor et al, 47 newly diagnosed hyperthyroid cats were used to investigate whether PLO formulated transdermal methimazole was safe and efficacious in controlling feline hyperthyroidism. At two weeks of treatment, more cats in the oral methimazole group had serum $\mathrm{T} 4$ concentrations within the reference range (14 of 16 [88\%], $\mathrm{p}=0.035$ ). By week four, there was no difference between the oral and transdermal methimazole. The PLO transdermal methimazole group took longer to reduce serum $\mathrm{T} 4$ concentrations to the acceptable reference range, however, it was as effective as oral administration in producing euthyroidism by the fourth week of treatment [30]. Fewer GI adverse events were reported with the transdermal formulation ( $1 / 27$ vs $4 / 17$ in the oral group). The reduction of GI upset deems consideration as it is often the cause of discontinuation of oral methimazole [30,31].

Lecuyer et al evaluated the efficacy of transdermal methimazole in 13 newly diagnosed hyperthyroid cats. The feline patients received $5 \mathrm{mg}$ methimazole concentrated in PLO, applied to the inner ear twice daily. In addition to reaching the euthyroid state, all 10 cats that completed the study also showed improved clinical signs related to hyperthyroidism consistent with other previously reported studies [16,32-33]. No GI adverse events were reported, and investigators concluded that PLO transdermal methimazole is a safe and effective alternative to oral methimazole [6].

\section{Duration of $\mathrm{t} 4$ suppression}

A study by Boretti et al. [33] evaluated the duration of serum T4 suppression among newly diagnosed hyperthyroid cats treated with once daily transdermal methimazole versus twice daily dosing. Twenty cats were treated with the PLO-based methimazole formulation dosed either $2.5 \mathrm{mg}$ every 12 hours (10 cats, group 1) or $5 \mathrm{mg}$ every 24 hours (10 cats, group 2). Serum T4 concentrations were measured one and three weeks after initiation of therapy, immediately before and every two hours after gel application for up to 10 hours. Cats were limited to a maximum of five blood samplings in one day [33]. A sustained suppression of $\mathrm{T} 4$ concentration for at least 24 hours was seen following gel application and there was no significant difference in change in serum T4 concentration immediately before or any time after gel administration in either group. As also discussed in Lecuyer's study [6], further research is needed concerning the duration of intra thyroid methimazole accumulation [6,33,34]. Among the twice daily dosing group, reductions were required in three cats, and a dose increase was required in one patient. Of the once daily dosing group, two cats required a decrease in dose, and one cat required an increased dose, after three weeks of treatment as a result of sustained hyperthyroid levels [33]. Investigators concluded that once daily application of the PLO methimazole compound can effectively reduce serum T4 concentrations in most hyperthyroid cats. Once a day dosing is most convenient for the owner, and thus promotes better compliance [33]. The compounding of this preparation allows for changes in dose or frequency and allows for the individualization of therapy.

\section{PLO vs. novel lipophilic base}

In a 12-week prospective study by Hill et al, a novel lipophilic formulation of methimazole was investigated. The study included 45 cats newly diagnosed with untreated, naturally occurring hyperthyroidism [12]. The study used a novel lipophilic formulation prepared with methimazole, "carrier compounds" (propylene glycol, polyethylene glycol 4000, dimethyl formamide, and cyclodextrin), and several penetration enhancers, chosen from fatty acids, terpenes, pyrrolidones, a short chain alcohol, glycol ethers, acetins, and triglycerides. The formulation was determined to be stable for 12 months after preparation, by the International Cooperation on Harmonization of Technical Requirements for Registration of Veterinary Products. Cats were treated with a starting dose of either oral carbimazole (5mg twice a day) or the novel transdermal methimazole formulation (10 mg, or $0.1 \mathrm{~mL}$ applied to the inner ear once a day). Both the once daily novel transdermal methimazole and twice daily oral carbimazole were effective in the treatment of feline hyperthyroidism in cats with compliant owners. All owners were satisfied with the improved clinical symptoms.

The novel lipophilic transdermal formulation had several advantages over the oral carbimazole, as the transdermal medication was tolerated better, and caused no gastrointestinal side effects in the cats. Owners reported that administering tablets to their cats was a challenge, and 35\% admitted to missing doses or cats spitting out the medication [12]. Unlike the rare occurrences of pruritus reported with the PLO formulation of methimazole, no adverse events of pruritus or erythema of the inner ear were reported $[6,12]$. The study suggests that since methimazole is a lipophilic drug, a lipophilic vehicle might more suitable than the PLO base.

Although this study clearly highlights the effectiveness of once a day use of this novel lipophilic formulation, it would have been more appropriate to study it in comparison with the PLO methimazole formulated topical. The novel lipophilic formulation appears to be less irritating to the skin among cats than the PLO. However, this has not been shown clinically significant in any study, and thus does not provide enough evidence to recommend one transdermal formulation over the other $[6,12,33]$. Further evaluation and study are needed to compare the costs, efficacy, stability, accessibility, and adverse event rates between the PLO and novel lipophilic formulations of methimazole.

\section{Discussion}

Transdermal drug delivery is an appealing route of administration for veterinary medicine, especially for clients with 
uncooperative pets. PLO used for methimazole is recognized as a viable transdermal delivery tool because of its enhanced drug transport capabilities. It can effectively deliver both hydrophilic and lipophilic drugs. Transdermal methimazole circumvents the liver's first pass metabolism, potentially allowing a lower drug dose for an equal effect while also avoiding the intolerable GI upset often caused by oral drugs leading to discontinuation. Following chronic daily application of PLO formulated methimazole to the inner ear of cats with hyperthyroidism, successful resolution of clinical signs and lower T4 levels have been noted $[6,18,30,31,33]$.

Although ultimately effective, delayed onset of action was noted and transdermal methimazole takes longer to achieve therapeutic serum T4 concentrations compared to oral methimazole activity. Oral administration may be more suitable in cats with very severe hyperthyroidism, requiring rapid reduction of thyroid hormone levels. Repeated dosing with the PLO formulation can lead to exfoliation of the inner ear, mild inflammation, and may cause a depot of drug in the skin [30,35]. As the PLO works to compromise the skin barrier over time, more drug is absorbed. Therefore, maximum effectiveness is not seen immediately, but most feline patients will reach a euthyroid level by week 4 of treatment. Transdermal methimazole can be deemed noninferior to the widely approved oral formulation.

Oral methimazole has only been proven effective if dosed twice a day in cats [16]. Once daily dosing of transdermal methimazole was successful, however, the need for twice daily dosing was recognized early in treatment. Once daily dosing presents an obvious advantage as it is most convenient for the owner and aids in promoting good compliance. Near perfect compliance is imperative when treating hyperthyroidism, because serum T4 concentrations can return to their hyperthyroid level within 48 hours after the last dose. Another unique advantage of the transdermal formulation is that it can be compounded into any dosage concentration needed.

In the past, transdermal methimazole was recommended only for short-term use in cases of oral methimazole induced GI upset or an uncooperative cat. Oral methimazole was indirectly favored due to the cost, variable stability, and unknown pharmacokinetic information of the transdermal form. However, more recent studies have suggested extended effectiveness with long-term use of the transdermal methimazole. Also, upon diagnosis of hyperthyroidism, most cats are near the end of the life and shortterm treatment is usually enough in resolving the hyperthyroid illness until the cat expires due to other unrelated diseases. Although the transdermal formulation is more expensive, it is still a more reasonable cost compared to the expense of thyroidectomy and radioactive therapy. Cat owners reported missing oral doses or cats spitting tablets, thus the transdermal gel may be worth the extra cost in order to manage the disease. Clients at large reported satisfaction with the compounded medicine, with only a few reports of precipitation of the gel [6].

\section{Conclusion}

Transdermal use of PLO compounded methimazole is an effective therapy for lowering serum T4 concentrations in cats. It is safe, posing fewer adverse effects than the oral formulation. It can be effectively used to treat feline hyperthyroidism through individualized dosing and frequency of administration. Owners should rotate ears each application and remove any residue with a damp cotton ball prior to the next application. Cats tolerate it very well, and it is favored by owners for its convenience and resolved GI upset events. Frequent monitoring of the cat's liver function tests, BUN, creatinine, $\mathrm{CBC}$, platelet count, and serum T4 concentration is recommended. Very little data exists regarding its pharmacokinetic properties and formulation stability, and the significance of the information available is limited by the small sample sizes studied.

\section{References}

1. Edinboro CH, Scott-Moncrieff JC, Janovitz E, Thacker HL, Glickman LT (2004) Epidemiologic study of relationships between consumption of commercial canned food and risk of hyperthyroidism in cats. J Am Vet Med Assoc 224(6): 879-886.

2. Trepanier LA (2007) Pharmacologic Management of Feline Hyperthyroidism. Vet Clin North Am Small Anim Pract 37(4): 775-788.

3. Peterson ME, Kintzer PP, Hurvitz AI (1988) Methimazole treatment of 262 cats with hyperthyroidism. J Vet Intern Med 2(3): 150-157.

4. Peterson ME (2010) Hyperthyroidism In: Ettinger SJ, Feldmand (Eds.), E.C. St. Louis Textbook of Veterinary Internal Medicine. $7^{\text {th }}$ (Edn), MO: Saunders Publishers, USA, pp. 1400-1417.

5. Feldman EC, Nelson RW (2004) Canine and Feline Endocrinology and Reproduction. ( $3^{\text {rd }}$ edn), Philadelphia, PA: Saunders Publishing, pp. 152-215.

6. Lecuyer M, Prini S, Dunn ME, Doucet MY (2006) Clinical efficacy and safety of transdermal methimazole in the treatment of feline hyperthyroidism. Can Vet J 47(2): 131-135.

7. Jonklaas J, Talbert RL (2015) Thyroid Disorders. In: Di Piro JT, Talbert RL, et al. (Eds.), Pharmacotherapy: A Pathophysiologic Approach. ( $9^{\text {th }}$ edn), Chapter 58, McGraw-Hill: New York, USA.

8. BrentGA, Koenig RJ (2011) Thyroid and Anti-Thyroid Drugs. In: Chabner BA, Knollmann BC (Eds.), Goodman \& Gilman's The Pharmacological Basis of Therapeutics. $\left(12^{\text {th }}\right.$ edn $)$, Chapter 39. McGraw-Hill: New York, USA.

9. Dong BJ, Greenspan FS (2012) Thyroid \& Antithyroid Drugs. In: Katzung BG, Masters SB, Trevor AJ (Eds.), Chapter 38, Basic \& Clinical Pharmacology, McGraw-Hill: New York, USA.

10. Bauer DC, McPhee SJ (2015) Thyroid Disease. In: Bauer DC, McPhee SJ (Eds.), Pathophysiology of Disease: An Introduction to Clinical Medicine. ( $6^{\text {th }}$ edn), Chapter 20, McGraw-Hill: New York, USA.

11. Trepanier LA (2006) Medical management of hyperthyroidism. Clin Tech Small Anim Pract 21(1): 22-28.

12. Hill KE, Gieseg MA, Kingsbury D, Lopez-Villalobos, N Bridges J, et al. (2011) The efficacy and safety of a novel lipophilic formulation of methimazole for the once daily transdermal treatment of cats with hyperthyroidism. J Vet Intern Med 25(6): 1357-1365.

13. Frenais R, Burgaud S, Horspool LJ (2008) Pharmacokinetics of controlled release carbimazole tablets support once daily dosing in cats. J Vet Pharmacol Ther 31(3):213-219. 
14. Chapman SF (2011) Homeopathic and integrative treatment for feline hyperthyroidism-four cases (2006-2010). Homeopathy 100(4): 270274.

15. Methimazole (2016) Lexi-Drugs Online. Lexi-Comp Inc, Hudson, Ohio, USA.

16. Trepanier LA, Hoffman SB, Kroll M, Rodan I, Challoner L (2003) Efficacy and safety of once versus twice daily administration of methimazole in cats with hyperthyroidism. J Am Vet Med Assoc 222(7): 954-958.

17. Trepanier L, Peterson M, Aucoin D (1991) Pharmacokinetics of methimazole in normal cats and cats with hyperthyroidism. Res Vet Sci 50(1): 69-74.

18. Murdan S (2005) A review of pluronic lecithin organogel as a topical and transdermal drug delivery system. Hospital Pharmacist 12: 267270.

19. Allen Loyd V (2003) The history of pluronic lecithin organogel: An Interview with Marty Jones. Int J Pharma Comp 7, 180-183.

20. Almeida H, Amarel MH, Lobao P, Lobo JM (2012) Pluronic F-127 and Pluronic Lecithin Organogel (PLO): Main Features and their Applications in Topical and Transdermal Administration of Drugs. J Pharm Pharm Sci 15(4): 592-605.

21. Agrawal V, Gupta V, Ramteke S, Trivedi P (2010) Preparation and evaluation of tubular micelles of pluronic lecithin organogel for transdermal delivery of sumatropin. AAPS Pharm SciTech 11(4): 17181725.

22. Patil KD (2011) Organogel: topical and transdermal drug delivery system. Int J Pharm Res Dev 3: 58-66.

23. Belgamwar VS, Pandey MS, Chauk DS, Surana J (2008) Pluronic lecithin organogel. Asian J Pharm 2: 134-138.

24. Boothe DM (2006) Transdermal gel drug therapy: fuss or must? (Proceedings) In: Boothe DM (Ed.), Veterinary Compounding in Small Animals: A Clinical Pharmacologist's Perspective In: Veterinary Clinics of North America USA.
25. Franckum J, Ramsay D, Das NG, Das SK (2004) Pluronic lecithin organogel for local delivery of anti-inflammatory drugs. Int J Pharm Compd 8(2): 101-105.

26. Kumar R, Katare OP (2005) Lecithin Organogels as a Potential Phospholipid-Structured System for Topical Drug Delivery: A Review. AAPS Pharm SciTech 6(2): E298-E310.

27. Riviere JE, Papich MG (2001) Potential and problems of developing transdermal patches for veterinary applications. Adv Drug Deliv Rev 50(3): 175-203.

28. Lusiana, Muller-Goyman CC (2011) Preparation, Characterization, and In Vitro Permeation Study of Terbinafine $\mathrm{HCl}$ in Poloxamer 407-Based Thermogelling Formulation for Topical Application. AAPS Pharm SciTech 12(2): 496-506.

29. Wingate G (2002) Transdermal methimazole in the treatment of 16 cats with hyperthyroidism. Int J Pharm Compd 6(5): 344-345.

30. Sartor LL, Trepanier LA, Kroll MM, Rodan I, Challoner L (2004) Efficacy and safety of transdermal methimazole in the treatment of cats with hyperthyroidism. J Vet Intern Med 18(5): 651-655.

31. Davidson GS (2005) Update on Transdermals for Animal Patients. Int J Pharm Compd 9(3): 178-182.

32. Hoffman SB, Yoder AR, Trepanier LA (2002) Bioavailability of transdermal methimazole in a pluronic lecithin organogel in healthy cats. J Vet Pharmacol Ther 25(3): 189-193.

33. Boretti FS, Sieber-Ruckstuhl NS, Schafer S, Baumgartner C, Riond B, et al. (2013) Duration of T4 suppression in hyperthyroid cats treated once and twice daily with transdermal methimazole. J Vet Intern Med 27(2): 377-381.

34. Jansson R, Dahlberg PA, Johansson H, Lindstrom B (1983) Intrathyroidal concentrations of methimazole in patients with Graves' disease. J Clin Endocrinol Metab 57(1): 129-132.

35. Deher F, Walde P, Luisi P, Elsner P (1996) Human skin irritation studies of a lecithin microemulsion gel and of lecithin liposomes. Skin Pharmacol 9(2): 124-129.

\section{Your next submission with Juniper Publishers will reach you the below assets}

- Quality Editorial service

- Swift Peer Review

- Reprints availability

- E-prints Service

- Manuscript Podcast for convenient understanding

- Global attainment for your research

- Manuscript accessibility in different formats

( Pdf, E-pub, Full Text, Audio)

- Unceasing customer service

Track the below URL for one-step submission

https://juniperpublishers.com/online-submission.php 